\title{
Ischemic Postconditioning and Subanesthetic S(+)-Ketamine Infusion: Effects on Renal Function and Histology in Rats
}

\author{
Marco A. C. de Resende, ${ }^{1,2}$ Alberto V. Pantoja, ${ }^{2}$ Bruno M. Barcellos, ${ }^{2}$ Eduardo P. Reis, ${ }^{3}$ \\ Thays D. Consolo, ${ }^{3}$ Renata P. Módolo, ${ }^{3}$ Maria A. C. Domingues, ${ }^{4}$ Alexandra R. Assad, ${ }^{2}$ \\ Ismar L. Cavalcanti, ${ }^{2}$ Yara M. M. Castiglia, ${ }^{5}$ and Norma S. P. Módolo ${ }^{5}$ \\ ${ }^{1}$ Pós-Graduação em Anestesiologia, Faculdade de Medicina de Botucatu, Universidade Estadual Paulista (UNESP), \\ 18618-970 Botucatu, SP, Brazil \\ ${ }^{2}$ Serviço de Anestesiologia, Departamento de Cirurgia, Universidade Federal Fluminense (UFF), 24033-900 Niterói, RJ, Brazil \\ ${ }^{3}$ Faculdade de Medicina de Botucatu, UNESP, 18618-970 Botucatu, SP, Brazil \\ ${ }^{4}$ Departamento de Patologia, UNESP, 18618-970 Botucatu, SP, Brazil \\ ${ }^{5}$ Departamento de Anestesiologia, UNESP, 18618-970 Botucatu, SP, Brazil
}

Correspondence should be addressed to Marco A. C. de Resende; macresende@gmail.com

Received 16 February 2015; Accepted 1 May 2015

Academic Editor: Ahmet Eroglu

Copyright (C) 2015 Marco A. C. de Resende et al. This is an open access article distributed under the Creative Commons Attribution License, which permits unrestricted use, distribution, and reproduction in any medium, provided the original work is properly cited.

Background. Ischemic postconditioning (IP) in renal Ischemia reperfusion injury (IRI) models improves renal function after IRI. Ketamine affords significant benefits against IRI-induced acute kidney injury (AKI). The present study investigated the effects of IP and IP associated with subanesthetic S(+)-ketamine in ischemia-reperfusion-induced AKI. Methods. Forty-one Wistar rats were randomized into four groups: CG (10), control; KG (10), S(+)-ketamine infusion; IPG (10), IP; and KIPG (11), S(+)-ketamine infusion + IP. All rats underwent right nephrectomy. IRI and IP were induced only in IPG and KIPG by left kidney arterial occlusion for $30 \mathrm{~min}$ followed by reperfusion for $24 \mathrm{~h}$. Complete reperfusion was preceded by three cycles of $2 \mathrm{~min}$ of reocclusion followed by $2 \mathrm{~min}$ of reperfusion. Renal function was assessed by measuring serum neutrophil gelatinase-associated lipocalin (NGAL), creatinine, and blood urea nitrogen (BUN). Tubular damage was evaluated by renal histology. Results. Creatinine and BUN were significantly increased. Severe tubular injury was only observed in the groups with IRI (IPG and KIPG), whereas no injury was observed in CG or KG. No significant differences were detected between IPG and KIPG. Conclusions. No synergic effect of the use of subanesthetic $\mathrm{S}(+)$-ketamine and IP on AKI was observed in this rat model.

\section{Introduction}

Ischemia-reperfusion injury (IRI) is an inevitable phenomenon during transplantation [1]. IRI is also one of the causes of delayed graft function and acute kidney injury (AKI) after transplantation and is associated with multiple processes, including inflammatory cascade activation, ion accumulation, free reactive oxygen species (ROS) formation, endothelial dysfunction, platelet aggregation with microembolization, and immune activation [2].

AKI incidence continues to increase despite the use of new biomarkers in clinical care to anticipate diagnosis and improve treatment [3]. Reducing IRI by creating resistance against IRI through preconditioning and postconditioning the organ has become an area of increasing interest. The principle of brief alternating cycles of arterial occlusion and reperfusion prior to complete reperfusion after a prolonged ischemic episode, so-called "ischemic postconditioning” (IP), in kidneys was recently reviewed for several animal models by van den Akker et al., demonstrating benefits with respect to organ damage and kidney function [1].

Cell injury induced by reactive oxygen species (ROS) is a determinant of IRI. Ketamine infusion appears to inhibit lipid peroxidation and the amount of ROS [4]. The addition of low-dose ketamine prior to induction [5] or continuous infusion of the $\mathrm{S}(+)$ isomer diminishes the responses of 
proinflammatory cytokines during and after cardiac surgery involving cardiopulmonary bypass [6]. Recently, evidence of the involvement of NMDA (N-methyl-D-aspartate) in AKI has been reported [7]. It has been demonstrated that AKI is associated with both the activation of NMDA receptors and significant oxidative stress. The antagonism of various allosteric sites of NMDA receptors, including the polyaminebinding site inhibitor ketamine, affords significant benefit against IR-induced AKI. Additionally, at low doses and with continuous infusion, the noncompetitive NMDA antagonist $\mathrm{S}(+)$-ketamine could minimize sympathomimetic action and present anti-inflammatory action.

The aim of this study was to investigate the effects of IP and IP associated with subanesthetic $S(+)$-ketamine on renal function and histology in rats.

\section{Materials and Methods}

2.1. Experimental Protocol. The rats received care that complied with the Guide for the Care and Use of Laboratory Animals published by the National Institutes of Health and with Brazilian law regarding animal experimentation. Following approval from the Research Ethics Committee for Animal Experimentation (protocol number 946/2012) of Botucatu Medical School, São Paulo State University (UNESP), 41 male Wistar rats weighing 300-500 g were allocated into four groups: CG, control group, $n=10 ; \mathrm{KG}$, subanesthetic $\mathrm{S}(+)$ ketamine continuous infusion, $n=10$; IPG, IP, $n=10$; and KIPG, subanesthetic S(+)-ketamine continuous infusion + IP, $n=11$. All rats were submitted to right nephrectomy. Postconditioned rats (IPG and KIPG) were also submitted to left kidney arterial occlusion for 30 minutes (min); complete reperfusion for 24 hours (h) was preceded by three cycles, $2 \mathrm{~min}$ of reperfusion followed by $2 \mathrm{~min}$ of reocclusion each, for a total of $12 \mathrm{~min}$. To achieve anesthesia inhalation, the rats were housed in a bell jar suitable for small animals. Anesthesia was initiated with $4 \%$ isoflurane (vaporizer, Ohmeda) with a total flow of $1 \mathrm{~L} \cdot \mathrm{min}^{-1}$ oxygen and $1 \mathrm{~L} \cdot \mathrm{min}^{-1}$ air. Once the rats could be manipulated, the isoflurane concentration was reduced to $2 \%$ and was administered by a nonrebreathing mask system under spontaneous respiration (Figure 1).

The temperature of the rats was maintained between $35.5^{\circ} \mathrm{C}$ and $37.5^{\circ} \mathrm{C}$ using a thermal blanket and was monitored with a rectal thermometer. The right internal jugular vein (RIJV) was dissected and cannulated with a $24 \mathrm{GA}$ venocath for infusion of Ringer lactate solution (RL) $\left(3 \mathrm{~mL} \cdot \mathrm{kg}^{-1} \cdot \mathrm{h}^{-1}\right)$ in all groups and subanesthetic $\left(1.25 \mathrm{mg} \cdot \mathrm{kg}^{-1} \cdot \mathrm{h}^{-1}\right) \mathrm{S}(+)-$ ketamine continuous infusion only in KG and KIPG, in compliance with the Food and Drug Administration (FDA) publication Guidance for Industry: Estimating the Maximum Safe Starting Dose in Initial Clinical Trials for Therapeutics in Adult Healthy Volunteers [8]. The left carotid artery (LCA) was dissected and cannulated with a $24 \mathrm{GA}$ venocath to monitor invasive arterial pressure through the transducer of a Datex-Engström recorder (Finland) and to obtain blood samples. In KG and KIPG, S(+)-ketamine drug infusion was initiated for $15 \mathrm{~min}$ before laparotomy and maintained until the end of the experiments. The following parameters were

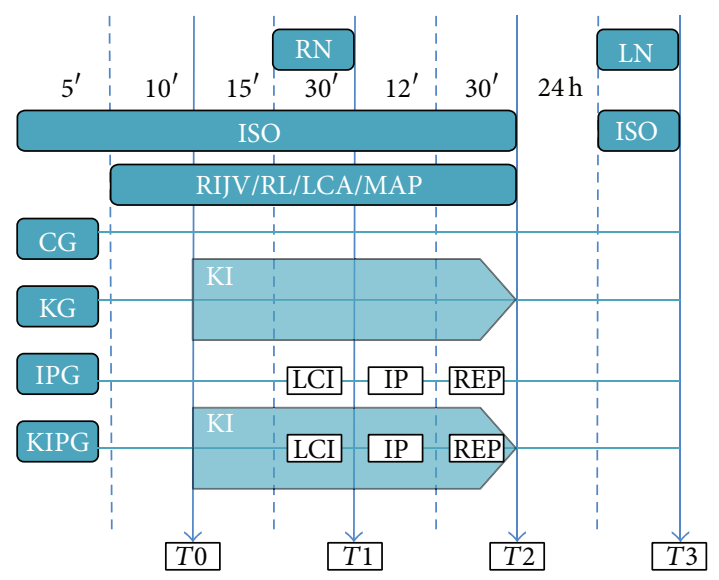

FIGURE 1: Experimental algorithm. ISO: isoflurane; RIJV: right internal jugular vein cannulation; RL: ringer lactate; LCA: left carotid artery; MAP: mean arterial pressure; RN; right kidney nephrectomy; LN: left kidney nephrectomy; KI: S(+)-ketamine infusion; LCI: left artery clamping; IP: ischemia-reperfusion cycles (12 min total); REP: full reperfusion; CG: control group; KG: S(+)-ketamine group; IPG: ischemic postconditioning group; KIPG: $\mathrm{S}(+)$-ketamine ischemic postconditioning group.

recorded: mean arterial pressure and rectal temperature at 15 min after the beginning of the anesthesia or after the cannulation of the RIJV and LCA (T0), after $30 \mathrm{~min}$ of left renal artery clamping (T1), and after $30 \mathrm{~min}$ of reperfusion and the cycles of IP (T2). The following biochemical variables were evaluated: serum neutrophil gelatinase-associated lipocalin (NGAL), creatinine, sodium, and blood urea nitrogen (BUN) at $T 0, T 2$, and after $24 \mathrm{~h}$ of reperfusion (T3). Each blood sample $(2 \mathrm{~mL})$ was accompanied by bolus infusion of $4 \mathrm{~mL}$ of Ringer lactate. Laparotomy was performed under cutaneous infiltration with $0.7 \mathrm{~mL}$ of $0.125 \%$ bupivacaine. To prevent compensation by the nonischemic kidney, right nephrectomy was performed in all groups, as proposed in Dobashi's modified method [9]. For arterial clamping, a temporary atraumatic Schwartz clip (Rs-5452, Roboz Surgical Instrument Co. Inc., Gaithersburg, MD, USA) was used. Following closure of the abdominal wall, the rat was awakened, taken to the animal center, and placed in an individual cage in a temperature-controlled environment with free access to food and water. Twenty-four hours later, the rats were submitted to laparotomy under anesthesia using the same technique. Left nephrectomy was performed, and the third arterial blood sample (T3) was collected directly from the heart. The rats were then immediately sacrificed by intracardiac injection of bupivacaine.

2.2. Renal Function Analysis. Serum NGAL (Rat NGAL ELISA Kit, Bioporto Diagnostics, Gentofte, Denmark), creatinine, and BUN (standard kits) were estimated to evaluate renal function. Serum sodium was used to evaluate hemodilution among the groups.

2.3. Histological Analysis. The left kidneys were processed for histological analysis. Once removed, the kidneys were 
TABLE 1: Descriptive and longitudinal analysis of the biochemical variables within each group.

\begin{tabular}{|c|c|c|c|c|c|c|c|c|c|c|c|}
\hline \multirow{2}{*}{$\begin{array}{l}\text { Collect } \\
\text { Group }\end{array}$} & \multicolumn{3}{|c|}{ T0 } & \multicolumn{3}{|c|}{$T 2$} & \multicolumn{3}{|c|}{ T3 } & \multirow{2}{*}{ Time effect ${ }^{\mathrm{a}}$} & \multirow{2}{*}{ Contrast analysis } \\
\hline & Mean & & SD & Mean & & SD & Mean & & SD & & \\
\hline \multicolumn{12}{|l|}{$\mathrm{Cr}$} \\
\hline CG & 0.430 & \pm & 0.142 & 0.620 & \pm & 0.193 & 0.550 & \pm & 0.108 & 0.021 & $T 0<(T 2=T 3)$ \\
\hline KG & 0.350 & \pm & 0.053 & 0.520 & \pm & 0.079 & 0.570 & \pm & 0.082 & 0.0001 & $T 0<(T 2=T 3)$ \\
\hline IPG & 0.390 & \pm & 0.074 & 0.820 & \pm & 0.274 & 2.60 & \pm & 1.67 & 0.0001 & $T 0<T 2<T 3$ \\
\hline KIPG & 0.418 & \pm & 0.117 & 0.964 & \pm & 0.112 & 3.26 & \pm & 1.83 & 0.0001 & $T 0<T 2<T 3$ \\
\hline \multicolumn{12}{|l|}{ NGAL } \\
\hline CG & 5.8 & \pm & 4.9 & 20.0 & \pm & 23.0 & 217.2 & \pm & 65.4 & 0.0001 & $T 0<T 2<T 3$ \\
\hline KG & 5.3 & \pm & 4.5 & 5.2 & \pm & 2.7 & 226.5 & \pm & 96.6 & 0.0001 & $(T 0=T 2)<T 3$ \\
\hline IPG & 10.1 & \pm & 10.4 & 37.7 & \pm & 41.3 & 343.6 & \pm & 50.6 & 0.0001 & $T 0<T 2<T 3$ \\
\hline KIPG & 20.8 & \pm & 19.9 & 66.0 & \pm & 104.9 & 374.0 & \pm & 17.7 & 0.0001 & $T 0<T 2<T 3$ \\
\hline \multicolumn{12}{|l|}{$\mathrm{Na}^{+}$} \\
\hline CG & 132.4 & \pm & 3.2 & 130.9 & \pm & 4.1 & 134.4 & \pm & 2.2 & 0.091 & \\
\hline KG & 130.1 & \pm & 4.0 & 132.9 & \pm & 1.7 & 133.8 & \pm & 3.3 & 0.025 & $T 0<(T 2=T 3)$ \\
\hline IPG & 128.5 & \pm & 2.8 & 128.2 & \pm & 2.4 & 128.6 & \pm & 1.9 & 0.90 & \\
\hline KIPG & 130.7 & \pm & 3.3 & 129.4 & \pm & 2.7 & 131.4 & \pm & 3.6 & 0.32 & \\
\hline \multicolumn{12}{|l|}{ BUN } \\
\hline CG & 55.0 & \pm & 14.8 & 63.7 & \pm & 16.3 & 56.3 & \pm & 11.6 & 0.17 & \\
\hline KG & 50.6 & \pm & 3.1 & 58.0 & \pm & 3.9 & 60.1 & \pm & 8.5 & 0.004 & $T 0<(T 2=T 3)$ \\
\hline IPG & 45.4 & \pm & 5.3 & 60.8 & \pm & 7.2 & 174.5 & \pm & 70.1 & 0.0001 & $T 0<T 2<T 3$ \\
\hline KIPG & 46.4 & \pm & 5.9 & 62.9 & \pm & 4.2 & 206.0 & \pm & 76.2 & 0.0001 & $T 0<T 2<T 3$ \\
\hline
\end{tabular}

SD: standard deviation; $\mathrm{Cr}$ : creatinine; NGAL: neutrophil gelatinase-associated lipocalin; $\mathrm{Na}^{+}$: sodium; $\mathrm{BUN}$ : blood urea nitrogen; CG: control group; KG: subanesthetic $S(+)$-ketamine continuous infusion group; IPG: ischemic postconditioning (IP) group; KIPG: subanesthetic S(+)-ketamine continuous infusion + IP group.

${ }^{a}$ ANOVA for repeated measures within each experimental group.

sectioned longitudinally and stored in separate vials. They were maintained in Duboscq-Brazil solution for the first $48 \mathrm{~h}$ and were then preserved in $70 \%$ ethanol. Histological sections were stained with hematoxylin-eosin. Histological analysis was performed by a single pathologist blinded to the origin of the groups studied. The scale described by Park et al. [10] was used to determine the histological severity of tubular cell injury, which is graded from 1 to 5 as follows: grade 0 , no lesions; grade 1, mild injury, less than $10 \%$ tubular cell necrosis; grade 2, mild to moderate injury, 10 to $25 \%$; grade 3 , moderate to severe injury, 25 to $50 \%$; grade 4 , severe injury, 50 to $75 \%$; and grade 5 , severe to very severe injury, greater than $75 \%$ tubular cell necrosis.

2.4. Statistical Analysis. All data are expressed as the means \pm standard deviations (SDs). Repeated measures analysis of variance (ANOVA) and the contrast test were used to assess the behavior of the biochemical and monitored variables for three periods within each experimental group. One-way repeated measures ANOVA was used to determine whether the evolution of the variables was different among the groups (interaction effect). In these analyses, logarithmic transformation (natural log) was applied to the data due to the lack of normality, according to the Kolmogorov-Smirnov test. The nonparametric Kruskal-Wallis ANOVA was used to compare body weight among the four groups. The nonparametric histological scores were analyzed between IPG and KIPG using the Mann-Whitney test. In all instances, values of $p<$ 0.05 were considered statistically significant. The statistical analysis was processed using SAS System version 6.11 for Windows (SAS Institute, Inc., Cary, NC, USA).

\section{Results}

The body weight values were similar for all groups $(p=0.43)$. One-way repeated measures ANOVA of the monitoring variables (mean arterial pressure and temperature) showed no significant effects due to the interaction $(p=0.18$ and $p=$ 0.30 , resp.). No functional impairment or histological injury was detected in CG and KG. Contrast analysis of repeated measures ANOVA of the biochemical variables showed that the values obtained at $T 0$ (basal) were significantly lower than at $T 2$ (with 30 min of reperfusion, after the cycles of IP) and T3 (after $24 \mathrm{~h}$ ) in CG and KG, and the increase in values was progressive and significant between $T 0, T 2$, and $T 3$ for IPG and KIPG (Table 1). No significant differences were detected between $T 2$ and $T 3$ values for CG and KG.

3.1. Effects of $S(+)$-Ketamine on Renal Morphology and Function. A subanesthetic dose of S(+)-ketamine alone did not alter the functional parameters evaluated or promote any detectable injury in the histological analysis (grade 0 , 
TABLE 2: Repeated measures ANOVA one factor and contrast analysis among the groups.

\begin{tabular}{|c|c|c|c|c|c|c|}
\hline & \multicolumn{2}{|c|}{ Main effect } & \multirow{2}{*}{ Interaction } & \multicolumn{3}{|c|}{ Contrast analysis among groups } \\
\hline & Group & Time & & Time point match & $p$ value & Commentaries \\
\hline \multirow{3}{*}{$\mathrm{Cr}$} & \multirow{3}{*}{0.0001} & \multirow{3}{*}{0.0001} & \multirow{3}{*}{0.0001} & $T 0 \times T 2$ & 0.034 & $(\mathrm{CG}=\mathrm{KG})<\mathrm{KIPG}, \mathrm{IPG}$ \\
\hline & & & & $T 0 \times T 3$ & 0.0001 & $(\mathrm{CG}=\mathrm{KG})<(\mathrm{IPG}=\mathrm{KIPG})$ \\
\hline & & & & $T 2 \times T 3$ & 0.0001 & $(\mathrm{CG}=\mathrm{KG})<(\mathrm{IPG}=\mathrm{KIPG})$ \\
\hline \multirow{3}{*}{ NGAL } & \multirow{3}{*}{0.0008} & \multirow{3}{*}{0.0001} & \multirow{3}{*}{0.081} & $T 0 \times T 2$ & 0.041 & $\mathrm{KG}<(\mathrm{CG}=\mathrm{IPG}=\mathrm{KIPG})$ \\
\hline & & & & $T 0 \times T 3$ & 0.54 & $\mathrm{CG}=\mathrm{KG}=\mathrm{IPG}=\mathrm{KIPG}$ \\
\hline & & & & $T 2 \times T 3$ & 0.11 & $\mathrm{CG}=\mathrm{KG}=\mathrm{IPG}=\mathrm{KIPG}$ \\
\hline \multirow{3}{*}{$\mathrm{Na}$} & \multirow{3}{*}{0.0001} & \multirow{3}{*}{0.014} & \multirow{3}{*}{0.13} & $T 0 \times T 2$ & 0.096 & $\mathrm{CG}=\mathrm{KG}=\mathrm{IPG}=\mathrm{KIPG}$ \\
\hline & & & & $T 0 \times T 3$ & 0.21 & $\mathrm{CG}=\mathrm{KG}=\mathrm{IPG}=\mathrm{KIPG}$ \\
\hline & & & & $T 2 \times T 3$ & 0.30 & $\mathrm{CG}=\mathrm{KG}=\mathrm{IPG}=\mathrm{KIPG}$ \\
\hline \multirow{3}{*}{ Ur } & \multirow{3}{*}{0.0001} & \multirow{3}{*}{0.0001} & \multirow{3}{*}{0.0001} & $T 0 \times T 2$ & 0.004 & $(\mathrm{CG}=\mathrm{KG})<(\mathrm{IPG}=\mathrm{KIPG})$ \\
\hline & & & & $T 0 \times T 3$ & 0.0001 & $(\mathrm{CG}=\mathrm{KG})<(\mathrm{IPG}=\mathrm{KIPG})$ \\
\hline & & & & $T 2 \times T 3$ & 0.0001 & $(\mathrm{CG}=\mathrm{KG})<(\mathrm{IPG}=\mathrm{KIPG})$ \\
\hline
\end{tabular}

Cr: creatinine; NGAL: neutrophil gelatinase-associated lipocalin; $\mathrm{Na}^{+}$: sodium; BUN: blood urea nitrogen; CG: control group; KG: subanesthetic $\mathrm{S}(+)$-ketamine continuous infusion; IPG: ischemic postconditioning (IP) group; KIPG: subanesthetic S(+)-ketamine continuous infusion + IP group.

Park et al.). Repeated measures ANOVA showed a significant variation for sodium only in KG. Despite performing nephrectomy, ketamine infusion did not worsen the evolution of the biochemical variables.

\subsection{Effects of Postconditioning on Renal Morphology and} Function. IP alone (PG) promoted progressive increases in all renal function parameters (creatinine, NGAL, and BUN), which exhibited statistically significant differences from the basal values (T0). Longitudinal contrast analysis of the variables within the group showed that $T 0<T 2<$ T3. However, one-way repeated measures ANOVA, which verifies whether the progression in values is different between the groups, showed interaction effects for creatinine $(p<$ $0.0001)$ and BUN $(p<0.0001)$. Contrast analysis between the groups verified that creatinine and BUN were very similar in IPG and KIPG (Table 2). All PG rats showed some degree of histological lesion, according to Park et al.

\subsection{Effects of $S(+)$-Ketamine and Postconditioning on Renal} Morphology and Function. The values of renal function parameters obtained for KIPG were greater than those obtained for IPG; however, longitudinal contrast analysis showed similar progressions in these values between these groups. Repeated measures ANOVA (time effect) for KIPG showed statistically significant values for creatinine, NGAL, and BUN. One-way repeated measures ANOVA showed that only creatinine and BUN were statistically significant regarding the interaction effect; contrast analysis confirmed that IPG and KIPG showed very similar progressions in values. As observed for the IPG rats, all KIPG rats demonstrated tubular injury during the histological analysis.

3.4. Histopathology. Since no lesions were detected in CG or KG, only IPG and KIPG were analyzed regarding differences in the degree of renal injury. No differences were observed between these groups $(p=0.63)$ (Figure 2).

\section{Discussion}

In our study, IP was unable to prevent structural renal tubular damage. Subanesthetic $S(+)$-ketamine showed no additional beneficial effects for IP but was also not responsible for worsening lesion scores.

The best animal model for IP is controversial. In 2007, using a mouse model, Szwarc et al. were the first to demonstrate that IP could prevent ischemic AKI [11]. In an overview, van den Akker et al. reviewed 13 studies on renal IRI and IP with different in vivo models, demonstrating improvements in renal function and histology in most studies. Two studies did not present benefits in renal function in IP: morphological analysis revealed reductions in fibroses, and terminal deoxynucleotidyl transferase dUTP nick end labeling (TUNEL) results were altered [1]. In our study, IP did not protect renal function and histology. A possible explanation is the algorithm used for IP, with longer periods of reocclusion $(2 \mathrm{~min}$ ) and reperfusion $(2 \mathrm{~min})$ of the renal artery, as opposed to most of the other studies that used seconds $(10,15,30,45$, and $60 \mathrm{~s})$ [1]. Besides the fact that the period of initial ischemia was short $(30 \mathrm{~min})$, the differences in the cycles of ischemia ( $2 \mathrm{~min}$ ) and reperfusion $(2 \mathrm{~min})$ in the IP period in our study were longer $[3 \times 2 \mathrm{~min}(12 \mathrm{~min})$ compared with $6 \times 10 \mathrm{~s}(1 \mathrm{~min})$ in rats]. This possibility may be supported by the findings of Jiang et al., who studied three different algorithm periods in IP in mongrel dogs, six cycles of $15 \mathrm{~s}$, six cycles of $30 \mathrm{~s}$, and three cycles of $60 \mathrm{~s}$, and reported significant differences among the three algorithms [12]. The effect of the smaller cycle ( six cycles of $15 \mathrm{~s}$ ) was the best. Despite this finding, Serviddio et al. used three algorithms with more prolonged periods of ischemia and reperfusion in IP in rats, 3 cycles of $5 \mathrm{~min}, 6$ cycles of $5 \mathrm{~min}$, and 12 cycles of $5 \mathrm{~min}$, and reported improved kidney function and less necrosis [13]. Unfortunately, the optimal IP algorithm remains unknown. Generally, in smaller animals with faster heartbeats, a shorter period of time can be used (5-10 s) compared with larger animals (30-60s) for IP 


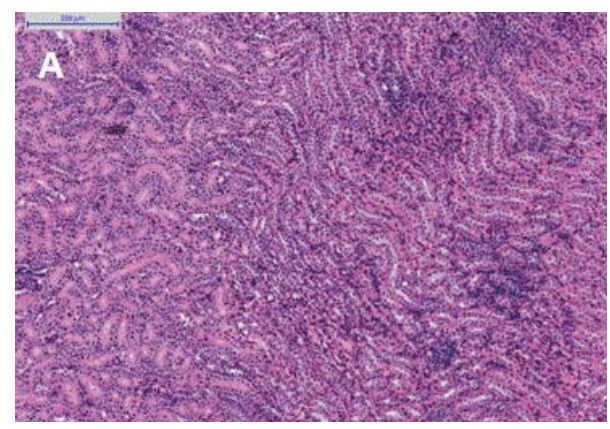

(a)

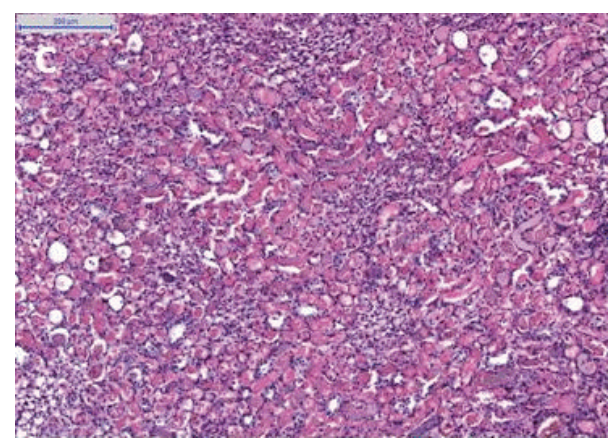

(c)

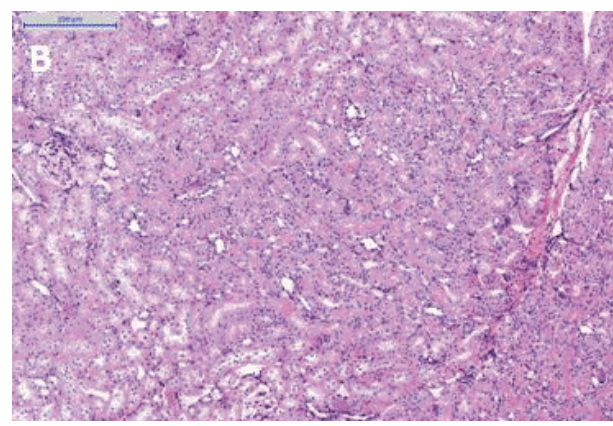

(b)

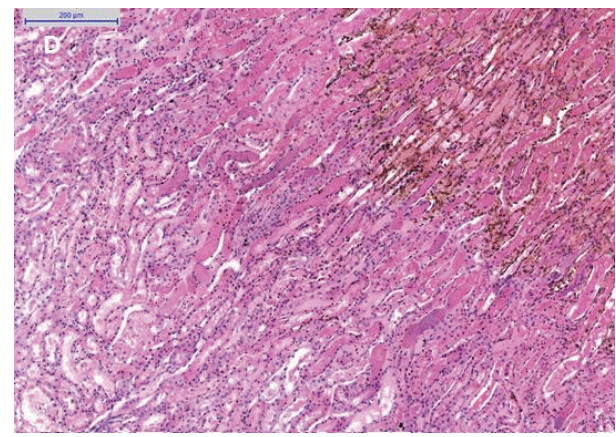

(d)

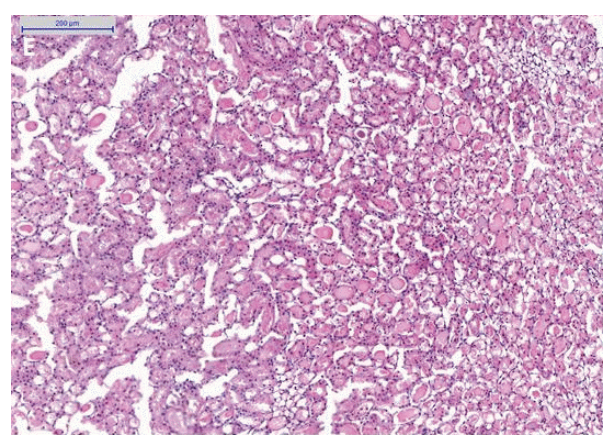

(e)

FIGURE 2: Representative light micrographs of rat kidneys, magnification 200x. Hematoxylin-eosin stain of kidney sections, graded for severity of tubular injury, according to Park et al. [10] (a) CG, left kidney, grade $0=$ no lesions; (b) KG, left kidney at 24 h, grade 0; (c) IPG, left kidney at $24 \mathrm{~h}$, grade 4 (severe injury); (d) IPG, left kidney at $24 \mathrm{~h}$, grade 5 (severe to very severe injury); (e) KIPG, left kidney at $24 \mathrm{~h}$, grade 4.

because of the correspondingly faster metabolic rate. The duration of ischemia is a determinant for the functional and histological severity of AKI; however, in the literature, we found differences in animal models, the duration of renal ischemia (30 to $90 \mathrm{~min}$ ), and the time between reperfusion and analysis ( 24 to $72 \mathrm{~h}$ ). In this study, we used a short period of ischemia ( $30 \mathrm{~min}$ ) because our period of cycles in IP was longer. Our results demonstrated that $30 \mathrm{~min}$ is sufficient to produce important renal damage in either morphology or function with significant elevations in serum Cr, BUN, and NGAL. Kidney transplantation is not ordinarily an elective procedure, and ischemic preconditioning cannot always be performed, but IP can be performed because it is a simple intervention. If IP is effective at reducing damage, questions arise, including the best model of IP and the possibility of translating IP results from animal studies to clinical practice in humans. The answers to these questions remain unclear.
In the context of renal transplantation, cold ischemia and the concomitant use of immunosuppressive medications could impair positive strategies for successful reperfusion. McCafferty et al. [14] requested a pause for thought because the potential benefit of IP, as reported in the article by van den Akker et al., may be ineffective at reducing reperfusion injury if the index ischemia has already caused irreversible injury. This possibility may also explain some degrees of lesions not measured in our study.

The N-methyl-D-aspartate receptor (NMDAR) is a heterotetrameric amino acid, part of the glutamate receptor family, originally described in the central nervous system, where it functions as a membrane calcium channel. These receptors definitely participate in various parts of the nephron, including the collecting ducts, glomerulus, and podocytes; therefore, it is not difficult to associate the receptors with a type of renal function regulation. In the neonatal kidney, 
NMDAR subunits NR3a and NR3b are expressed; Sproul et al. reported the upregulation of NR3a secondary to hypoxia and hypertonicity in a mouse model [15]. NMDARs promote renal vasodilator effects through the nitric oxide pathway [16]. However, NMDAR-mediated activation is associated with enhanced oxidative stress and renal damage, as observed in gentamicin renal injury or hyperhomocysteinemic rats [17, 18]. Antagonism of multiple-target sites of NMDAR seems to show benefits against AKI [7].

Ketamine is a noncompetitive antagonist of the NMDAR that binds at the polyamine-binding site. The protective role of ketamine through NMDAR blockade has been studied in the brain with additional regenerative effects for $\mathrm{S}(+)$ ketamine in cultured neurons [19]. In skeletal muscle, racemic ketamine at a subanesthetic dose has already been described as displaying beneficial effects in IRI [20]. Other NMDAR antagonists have been studied in renal IRI. In 2008, Yang et al. observed improvement in renal dysfunction following ischemia-reperfusion in rats with prior use of an NMDAR antagonist, D-2-amino-5-phosphopentanoic acid (D-AP-5) [21]. In acute tubular necrosis (ATN) promoted by gentamicin, in which overexpression of the NMDAR was observed, another antagonist of the NMDAR, MK-801, also promoted renal protection [18]. Recently Pundir et al. used a rat model of $40 \mathrm{~min}$ of bilateral renal ischemia followed by $24 \mathrm{~h}$ reperfusion and observed significant protection with ketamine in renal IRI at a significantly higher dose than that used for anesthesia [7]. A subanesthetic dose of $S(+)$ ketamine had no protective effect on the kidney in this study and did not improve or aggravate the renal damage in IP rats. One argument is that the subanesthetic dose of ketamine that we used may be insufficient to confer protection in this model of renal postconditioning, but the presence of structural lesions in the groups submitted to ischemia may have masked the possible benefits for functional damage. While the effects of NMDAR antagonists on the modulation of glomerular tone are still not fully understood, elevated serum levels of catecholamines, a dose-dependent response to ketamine administration, has a negative role in renal function, supporting our choice of a low-dose regimen with the more potent isomer. The highest scores for tubular lesions have been attributed to the use of $S(+)$-ketamine in rats associated with higher levels of catecholamines [22]. The use of subanesthetic doses preserves anti-inflammatory activity while reducing sympathomimetic action [23].

Serum creatinine, BUN, and NGAL are widely accepted for assessing renal function and renal histology reveals any parenchymal injuries. Creatinine is the most commonly used biomarker in clinical practice, even though NGAL presents greater sensitivity for the early detection of AKI [1]. Despite the limitations that body weight, mass, and hydration exert on creatinine, our results were consistent with a $24 \mathrm{~h}$ ischemic aggression protocol in the groups in which ischemia-reperfusion was performed. In this study, the histological evidence of renal injury corresponded with plasma levels of creatinine and urea. However, functional damage occurred even in the groups not submitted to ischemia (CG and $\mathrm{KG}$ ), as observed by the increase in NGAL, which was virtually unaltered between $T 0$ and $T 2$ (KG), but showed similarities with the remaining groups. The increase in NGAL is also considered to be secondary to comorbidities and systemic inflammation, which can be caused by surgical procedures. This biomarker is considered highly sensitive for discriminating AKI, but in this study, its expression was unexpectedly variable and inconclusive $[1,24]$. In this work, $\mathrm{S}(+)$ ketamine reduced the functional lesion, as demonstrated by NGAL values, in rats not submitted to the IP protocol. This effect most likely occurred because the drug attenuated inflammation in the right nephrectomy. Unfortunately, in our experimental IP model, we observed structural damage likely secondary to ischemia time, which did not allow the observation of a potential protective effect of the drug in this setting.

Evaluations of the weight of the rats and the control of blood pressure, temperature, and hydration were all adequately performed in our model; the groups exhibited homogeneous behaviors.

The majority of murine studies focus on AKI following bilateral warm ischemia [25]. Evolution to ATN and interstitial inflammatory infiltrate is characteristic in the first $24 \mathrm{~h}$ after reperfusion.

The mechanisms involved in pharmacological preconditioning and IP require experimental research that corroborates and improves our current understanding of the intervention models. Reflections concerning the results obtained accrue from trying to determine the optimal algorithm, from control of the intervention and from safe and favorable reproducibility, which permit clear extrapolation to clinical practice.

\section{Conclusion}

In our study, the effect of postconditioning itself was unable to prevent severe structural tubular injury, probably due to the prolonged ischemia time in the algorithm. We conclude that a subanesthetic dose of $\mathrm{S}(+)$-ketamine provided no additional beneficial effects for the postconditioning model, but neither was it responsible for the worst injury scores. The distinction in lesion progression between functional injury and permanent structural damage and how to anticipate and disrupt this complex process remains the subject of future research.

\section{Abbreviations}

AKI: Acute kidney injury

ANOVA: Analysis of variance

ATN: Acute tubular necrosis

BUN: Blood urea nitrogen

CG: $\quad$ Control group

FDA: Food and Drug Administration

IP: Ischemic postconditioning

IPG: Ischemic postconditioning group

IRI: Ischemia-reperfusion injury

KG: $\quad$ Subanesthetic S(+)-ketamine group

KIPG: Subanesthetic S(+)-ketamine ischemic postconditioning group

LCA: Left carotid artery 
T0: $\quad 15$ minutes after the beginning of the anesthesia

T1: $\quad$ After 30 min of left renal artery clamping

T2: After $30 \mathrm{~min}$ of reperfusion and cycles of ischemic postconditioning

T3: $\quad$ After $24 \mathrm{~h}$ of reperfusion

NGAL: Neutrophil gelatinase-associated lipocalin

NMDA: N-Methyl-D-aspartate

NMDAR: N-Methyl-D-aspartate receptor

RIJV: $\quad$ Right internal jugular vein

RL: $\quad$ Ringer lactate

ROS: $\quad$ Reactive oxygen species.

\section{Authors' Contribution}

Marco A. C. de Resende was responsible for acquisition and interpretation of data and was involved in experimental procedures and paper writing. Alberto V. Pantoja and Bruno M. Barcellos were responsible for statistical analysis. Eduardo P. Reis, Thays D. Consolo, and Renata P. Módolo were responsible for acquisition of data and helped with technical procedures. Maria A. C. Domingues was responsible for histopathological examinations. Alexandra R. Assad and Ismar L. Cavalcanti were responsible for paper preparation and critical revision. Yara M. M. Castiglia was responsible for study design and critical revision. Norma S. P. Módolo was responsible for study design, data analysis, and paper preparation and supervised all phases of study.

\section{Conflict of Interests}

The authors declare no conflict of interests.

\section{Acknowledgment}

This study was supported by the São Paulo Research Foundation (FAPESP), 2012/13606-1.

\section{References}

[1] E. K. van den Akker, O. C. Manintveld, D. A. Hesselink, R. W. F. de Bruin, J. N. M. Ijzermans, and F. J. M. F. Dor, "Protection against renal ischemia-reperfusion injury by ischemic postconditioning," Transplantation, vol. 95, no. 11, pp. 1299-1305, 2013.

[2] H. K. Eltzschig and T. Eckle, "Ischemia and reperfusion-from mechanism to translation," Nature Medicine, vol. 17, no. 11, pp. 1391-1401, 2011.

[3] E. D. Siew, "Blind men and elephants and the biological markers of AKI," Journal of the American Society of Nephrology, vol. 22, no. 9, pp. 1578-1580, 2011.

[4] A. E. Salman, D. Dal, M. A. Salman, A. B. Iskit, and Ü. Aypar, "The effect of ketamine on acute muscular ischaemia reperfusion in rats," European Journal of Anaesthesiology, vol. 22, no. 9, pp. 712-716, 2005.

[5] B. Beilin, Y. Rusabrov, Y. Shapira et al., "Low-dose ketamine affects immune responses in humans during the early postoperative period," British Journal of Anaesthesia, vol. 99, no. 4, pp. 522-527, 2007.

[6] I. D. Welters, M.-K. Feurer, V. Preiss et al., "Continuous S-(+)ketamine administration during elective coronary artery bypass graft surgery attenuates pro-inflammatory cytokine response during and after cardiopulmonary bypass," British Journal of Anaesthesia, vol. 106, no. 2, pp. 172-179, 2011.

[7] M. Pundir, S. Arora, T. Kaur, R. Singh, and A. P. Singh, "Effect of modulating the allosteric sites of N-methyl-D-aspartate receptors in ischemia-reperfusion induced acute kidney injury," Journal of Surgical Research, vol. 183, no. 2, pp. 668-677, 2013.

[8] Food and Drug Administration, Guidance for Industry: Estimating the Maximum Safe Starting Dose in Initial Clinical Trials for Therapeutics in Adult Healthy Volunteers, Center for Drug Evaluation and Research, US Department of Health and Human Services, Washington, DC, USA, 2005.

[9] K. Dobashi, I. Singh, J. K. Orak, K. Asayama, and A. K. Singh, "Combination therapy of $\mathrm{N}$-acetylcysteine, sodium nitroprusside and phosphoramidon attenuates ischemia-reperfusion injury in rat kidney," Molecular and Cellular Biochemistry, vol. 240, no. 1-2, pp. 9-17, 2002.

[10] Y. Park, R. Hirose, K. Dang et al., "Increased severity of renal ischemia-reperfusion injury with venous clamping compared to arterial clamping in a rat model," Surgery, vol. 143, no. 2, pp. 243251, 2008.

[11] I. Szwarc, S. Soullier, N. Gayrard, C. Mejean, G. Mourad, and A. Argiles, "Ischemic postconditioning prevents ischemic acute renal failure," Transplantation Proceedings, vol. 39, no. 8, pp. 2554-2556, 2007.

[12] B. Jiang, X. Liu, H. Chen et al., "Ischemic postconditioning attenuates renal ischemic/reperfusion injury in mongrel dogs," Urology, vol. 76, no. 6, pp. 1519.el-1519.e7, 2010.

[13] G. Serviddio, A. D. Romano, L. Gesualdo et al., "Postconditioning is an effective strategy to reduce renal ischaemia/reperfusion injury," Nephrology Dialysis Transplantation, vol. 23, no. 5, pp. 1504-1512, 2008.

[14] K. McCafferty, C. J. Byrne, and M. M. Yaqoob, "Renal postconditioning...pause for thought? Correspondence regarding 'protection against renal ischemia-reperfusion injury by ischemic postconditioning,' Transplantation, vol. 96, no. 7, pp. e51-e53, 2013.

[15] A. Sproul, S. L. Steele, T. L. Thai et al., "N-methyl-d-aspartate receptor subunit NR3a expression and function in principal cells of the collecting duct," The American Journal of Physiology-Renal Physiology, vol. 301, no. 1, pp. F44-F54, 2011.

[16] A. Deng, J. M. Valdivielso, K. A. Munger, R. C. Blantz, and S. C. Thomson, "Vasodilatory N-methyl-D-aspartate receptors are constitutively expressed in rat kidney," Journal of the American Society of Nephrology, vol. 13, no. 5, pp. 1381-1384, 2002.

[17] C. Zhang, F. Yi, M. Xia et al., "NMDA receptor-mediated activation of NADPH oxidase and glomerulosclerosis in hyperhomocysteinemic rats," Antioxidants and Redox Signaling, vol. 13, no. 7, pp. 975-986, 2010.

[18] J. C. Leung, T. Marphis, R. D. Craver, and D. M. Silverstein, "Altered NMDA receptor expression in renal toxicity: protection with a receptor antagonist," Kidney International, vol. 66, no. 1, pp. 167-176, 2004.

[19] S. Himmelseher, E. Pfenninger, and M. Georgieff, "The effects of ketamine-isomers on neuronal injury and regeneration in rat hippocampal neurons," Anesthesia and Analgesia, vol. 83, no. 3, pp. 505-512, 1996.

[20] Y. Ergün, H. Öksüz, Y. Atli, M. Kilinç, and S. Darendeli, "Ischemia-reperfusion injury in skeletal muscle: comparison of the effects of subanesthetic doses of ketamine, propofol, and etomidat," Journal of Surgical Research, vol. 159, no. 1, pp. el-e10, 2010. 
[21] C.-C. Yang, C.-T. Chien, M.-H. Wu, M.-C. Ma, and C.-F. Chen, "NMDA receptor blocker ameliorates ischemia-reperfusioninduced renal dysfunction in rat kidneys," American Journal of Physiology-Renal Physiology, vol. 294, no. 6, pp. F1433-F1440, 2008.

[22] E. R. Neto, P. T. Galvão Vianna, R. M. Viero et al., "Influence of $\mathrm{S}(+)$-ketamine analgesia in renal intraoperative ischemia. Histological study in rats," Acta Cirurgica Brasileira, vol. 21, no. 4, pp. 242-246, 2006.

[23] S. Himmelseher and M. E. Durieux, "Ketamine for perioperative pain management," Anesthesiology, vol. 102, no. 1, pp. 211220, 2005.

[24] E. MacEdo and R. L. Mehta, "Biomarkers for acute kidney injury: combining the new silver with the old gold," Nephrology Dialysis Transplantation, vol. 28, no. 5, pp. 1064-1067, 2013.

[25] S. E. Kennedy and J. H. Erlich, "Murine renal ischaemiareperfusion injury," Nephrology, vol. 13, no. 5, pp. 390-396, 2008. 


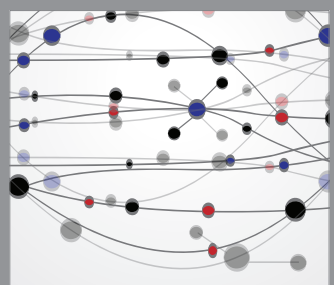

The Scientific World Journal
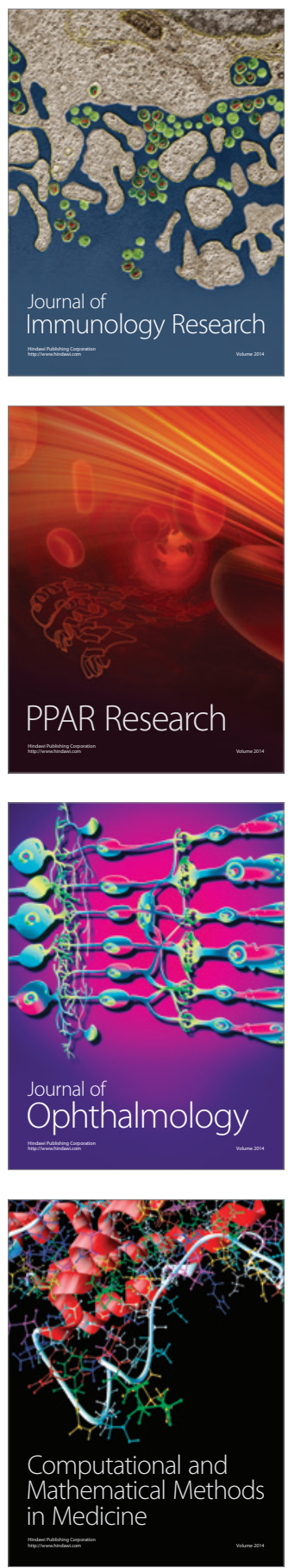

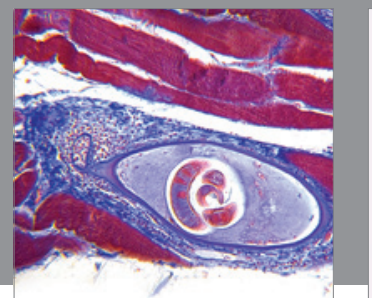

Gastroenterology

Research and Practice
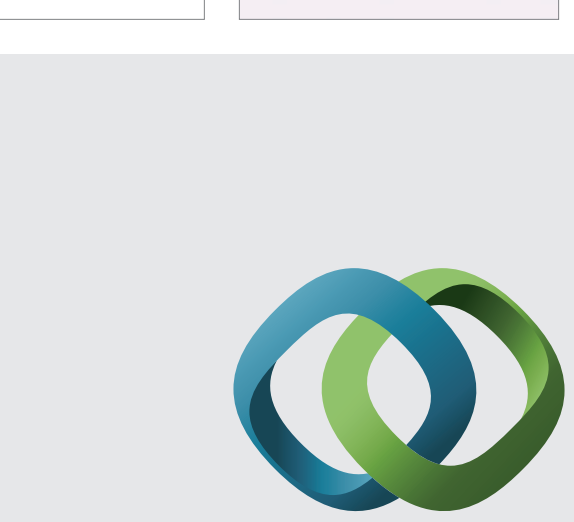

\section{Hindawi}

Submit your manuscripts at

http://www.hindawi.com
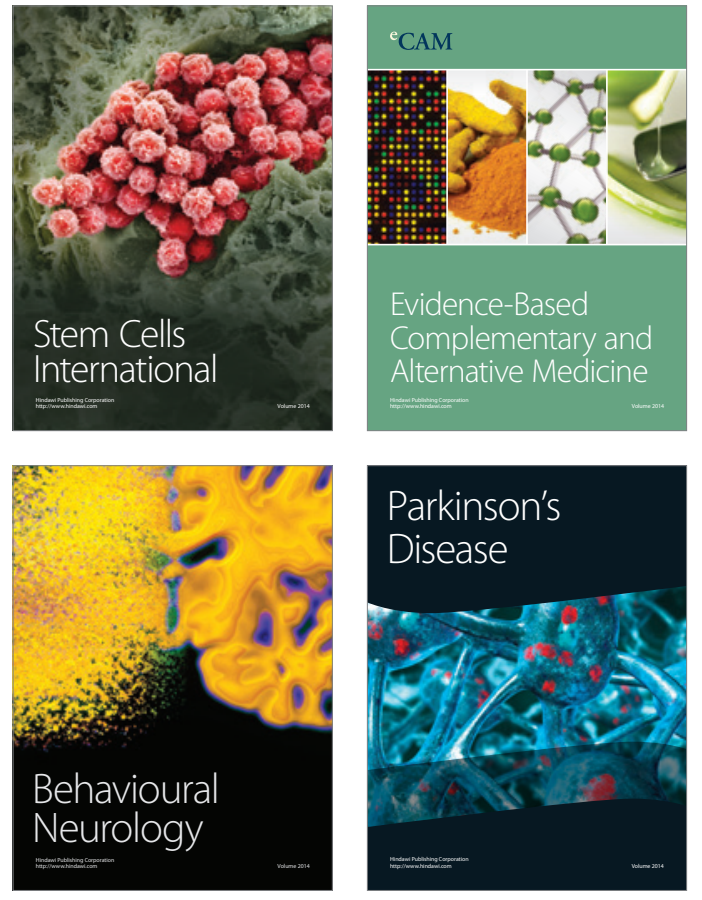
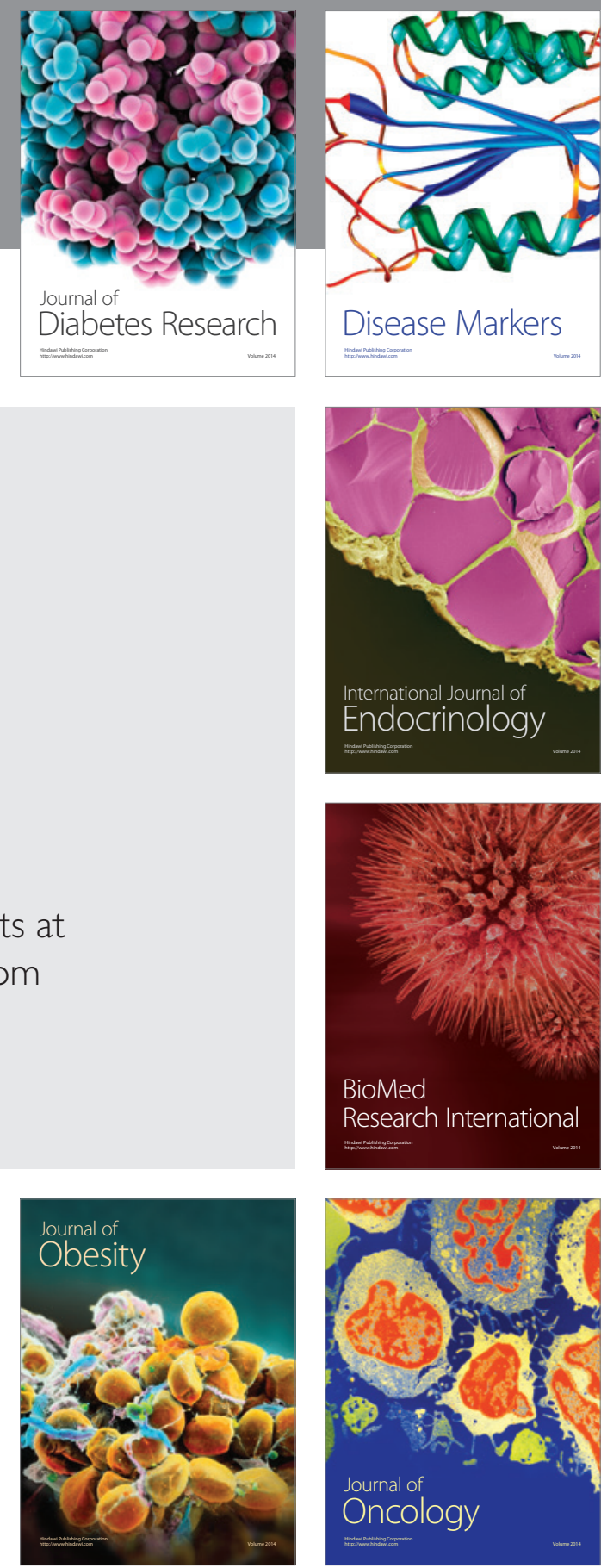

Disease Markers
